
\title{
BReserch S Suare \\ The Vibration Study of A Sandwich Conical Shell With A Saturated FGP Core
}

\author{
Mohsen Nasr Esfahani \\ Islamic Azad University \\ Mohammad Hashemian ( $\sim$ Hashemian@iaukhsh.ac.ir ) \\ Islamic Azad University \\ Farshid Aghadavoudi \\ Islamic Azad University
}

\section{Research Article}

Keywords: Free vibration, Conical shell, Sandwich shell, Porous materials, Biot's theory.

Posted Date: December 8th, 2021

DOI: https://doi.org/10.21203/rs.3.rs-1124240/v1

License: (1) This work is licensed under a Creative Commons Attribution 4.0 International License.

Read Full License 


\title{
The vibration study of a sandwich conical shell with a saturated FGP core
}

\author{
Mohsen Nasr Esfahani', Mohammad Hashemian ${ }^{1 *}$, Farshid Aghadavoudi ${ }^{1}$ \\ ${ }^{1}$ Department of Mechanical Engineering, Khomeinishahr Branch, Islamic Azad University, \\ Khomeinishahr, Isfahan, Iran \\ "Corresponding author: \\ Mohammad Hashemian \\ Department of Mechanical Engineering, Islamic Azad University, Khomeinishahr Branch, \\ Khomeinishahr 84175-119, Iran. \\ Emails: $\underline{\text { Hashemian@iaukhsh.ac.ir }}$
}

\begin{abstract}
This paper is provided to analyze the free vibration of a sandwich truncated conical shell with a saturated functionally graded porous (FGP) core and two same homogenous isotropic face sheets. The mechanical behavior of the saturated FGP is assumed based on Biot's theory, the shell is modeled via the first-order shear deformation theory (FSDT), and the governing equations and boundary conditions are derived utilizing Hamilton's principle. Three different porosity distribution patterns are studied including one homogenous uniform distribution pattern and two non-homogenous symmetric ones. The porosity parameters in mentioned distribution patterns are regulated to make them the same in the shell's mass. The equations of motion are solved exactly in the circumferential direction via proper sinusoidal and cosinusoidal functions, and a numerical solution is provided in the meridional direction utilizing the differential quadrature method (DQM). The precision of the model is approved and the influences of several parameters such as circumferential wave number, the thickness of the FGP core, porosity parameter, porosity distribution pattern, the compressibility of the pore fluid, and boundary conditions on the shell's natural frequencies are investigated. It is shown that the highest natural frequencies usually can be achieved when the larger pores are located close to the shell's middle surface and in each vibrational mode, there is a special value of the porosity parameter which leads to the lowest natural frequencies. It is deduced that in most cases, natural frequencies decrease by increasing the thickness of the FGP core. In addition, reducing the compressibility of the porefluid a small growth in the natural frequencies can be seen.
\end{abstract}

Keywords: Free vibration; Conical shell; Sandwich shell; Porous materials; Biot's theory. 


\section{Introduction}

Due to the numerous use of the conical shells in different engineering applications such as aerospace and mechanical engineering, high-power aircraft jet engines, high-speed centrifugal separators, and gas turbines, a considerable number of investigations have been presented on the mechanical analysis of such structures, recently. Sofiyev [1] investigated the stability and free vibration analyses of heterogenous composite truncated conical shells reinforced with carbon nanotubes (CNTs) subjected to an axial load. He examined the effects of the percentage of the CNTs and heterogeneity on the buckling and free vibrational characteristics of the shell. The free vibration characteristics of rotating polymeric truncated conical shells enriched by graphene nanoplatelets (GNPs) were investigated by Afshari [2]. It was shown by him that the sequence of vibrational modes can be affected by the variation of semi-vertex angle. By utilizing analytical and numerical techniques and experimental tests, the free vibration study of conical shells stiffened by bevel stiffeners was examined by Zarei et al. [3]. They studied the influences of the shell's geometrical characteristics on the natural frequencies of such a structure. Yousefi et al. [4, 5] studied the forced and free vibrational behavior of three-phase CNT/polymer/fiber truncated conical panels and shells. It was revealed by them that the larger length and higher embracing and semi-vertex angles result in the smaller natural frequencies. To complete these works, they hired particle swarm optimization to find the best values of mass fractions of the CNTs and fibers and orientation of the fibers to minimize the cost and maximize the fundamental frequency of the threephase CNT/polymer/fiber laminated truncated conical panels [6]. Aris and Ahmadi [7] studied the analysis of the nonlinear resonance of FGM (functionally graded materials) truncated conical shells exposed to an external harmonic excitation and thermal loading. They examined the effects of the shell's geometrical characteristics and temperature on the nonlinear vibrational characteristics of the shell. By incorporating the agglomeration of the CNTs, the free vibration study of a CNT-reinforced spinning truncated conical shell was examined by Afshari and Amirabadi [8]. It was shown by them that variation of the rotational speed may change the sequence of the vibrational modes. The vibration study of combined conical-ribbed cylindricalconical shell structures was investigated by Zhang et al. [10]. They approved the precision of their work by comparing their results with the corresponding ones obtained via the finite element method (FEM) and experiment tests. Fares et al. [11] employed a layerwise formulation and analyzed the free vibration of multilayered CNT-reinforced truncated conical shells. They checked the dependency of the natural frequencies on the thickness stretching strains. For various boundary conditions, the natural frequencies of porous metal foam truncated conical shells were reported by Li et al. [12]. They examined the effects of the porosity parameter and pore dispersion pattern on the shell's natural frequencies. Using FEM, Singha et al. [13] analyzed the free vibration of rotating 
pre-twisted sandwich conical shells with a homogenous core and FG graphene-reinforced face sheets in a thermal environment. They studied the effect of graphene distribution patterns on natural frequencies. Adab et al. [14] investigated the free vibrational behavior of spinning sandwich truncated conical microshells with an FGP core and GNP-reinforced face sheets. It was shown by them that the highest natural frequencies can be achieved when the big pores are located close to the middle surface of the microshell. Nasution et al. [15] succeeded to find a semianalytical solution for the supersonic flutter behavior of three-phase polymer/GNP/fiber laminated joined conical-conical shells. They concluded that the aeroelastic stability and flutter mode of such structures can be easily affected by the semi-vertex angles and lengths of the shell segments.

Due to some superior properties such as low density, high capacity of energy loss, low thermal conductivity, and high recyclability, porous materials have been receiving great interest as engineering materials in the transportation industry and mechanical, civil, and aerospace engineering [16]. The porous materials consist of two phases: the main phase is solid and the other one is either gas or liquid which can be found in nature such as layers of dust, stone, and wood [17]. The initial researches on the mechanical characteristics of porous materials were performed by Biot [18-21] when he proposed the constitutive relations in porous mediums known as Biot's theory. Some authors studied the mechanical analysis of the beams, plates, shells, and panels made of porous materials or the sandwich structures with an FGP core. Leclaire et al. [22] analyzed the dynamics of porous plates and studied the influences of porosity parameter and compressibility of pore fluid on the natural frequencies of such a plate. Kiani [24] provided the dynamic response of porous beams subjected to the action of a moving load. The influences of the speed of moving load, length to thickness ratio of the beam, and compressibility of pore fluid on the vibration amplitude were examined by him. Xiang et al. [25] investigated the forced vibrational behavior of thin rectangular porous plates. For various types of excitation, two selected boundary conditions, and two selected pore distribution patterns, they provided the vibration amplitude response versus excitation frequency. The mechanical buckling, static bending, and free vibration analyses of FGporous beams were studied by Fouda et al. [26]. They proposed two models to calculate the mechanical properties of the porous beam including an implicit model and an explicit one and investigated the dependencies of the mechanical characteristics (static deflection, natural frequencies, and critical buckling load) on the porosity parameter. Mojahedin et al. [27] studied the thermoelastic response of porous beams. They studied the dependency of the thermoelastic characteristics of such beams on the pore fluid. An exact solution was presented by Nikkhoo et al. [28] for the dynamic response of flexo-poro-elastic structures subjected to moving loads. The influences of the porosity parameter, viscosity of the pore fluid, the velocity of the moving loads, and stiffness of the foundation on the dynamic response of the beam were examined by them. Enayat et al. [29, 30] studied the mechanical buckling, static bending, and free vibration 
characteristics of porous nanobeams subjected to thermal loading. It was shown by them that by increasing the porosity parameter, the static deflection increases and the critical buckling load decrease; but the effect of the porosity parameter on the natural frequencies significantly depends on the pore distribution pattern. The free vibration and aeroelastic stability behaviors of sandwich cylindrical thick panels with saturated FGP core were examined by Akbari et al. [31, 32]. It was shown by them that the higher porosity parameter results in the weaker aeroelastic stability of the panel. The free vibration and aeroelastic stability behaviors of GNP-reinforced porous cylindrical panels were investigated by Zhou et al. [33]. They deduced that the critical speed of the panel diminishes by increasing the porosity parameter.

To the author's best knowledge, the presented paper is the first attempt to analyze the free vibration of sandwich truncated conical shells with two isotropic homogenous face sheets and a saturated FGP core. The dependencies of the natural frequencies on the circumferential wave number, boundary conditions, compressibility of the pore fluid, porosity distribution pattern, porosity parameter, and the thickness of the FGP core are examined.

\section{Mathematical modeling}

\subsection{Geometrical descripton}

As Fig. 1 shows, a sandwich truncated conical shell is considered of thickness $h$, small radius $a$, length $L$, semi-vertex angle $\alpha$, and large radius $b=a+L \sin \alpha$. The shell consists of a saturated FGP core of thickness $h_{\mathrm{c}}$ and two same homogenous isotropic face sheets of thickness $h_{\mathrm{f}}=0.5\left(h-h_{\mathrm{c}}\right)$.

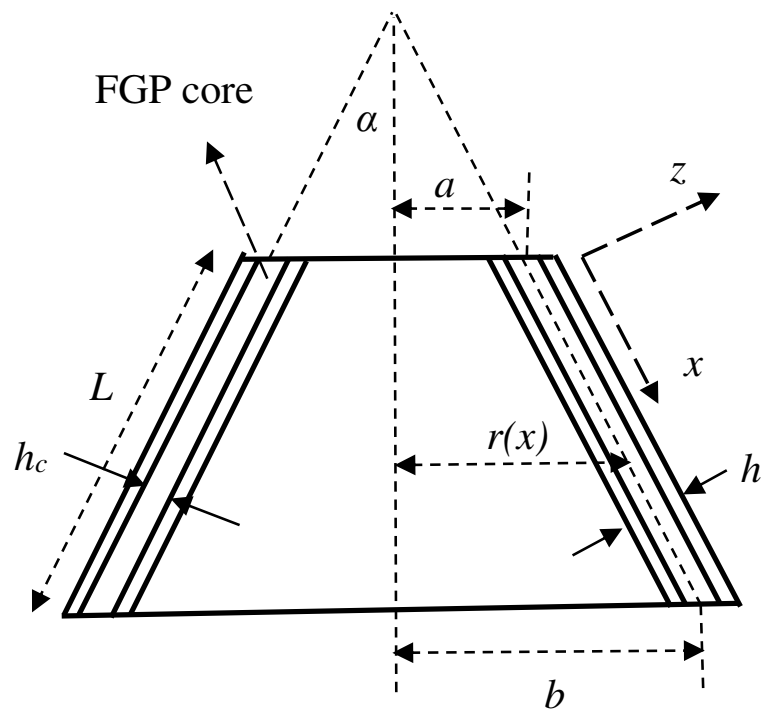

Fig. 1. A sandwich truncated conical shell with an FGP core 


\subsection{Material properties}

As depicted in Fig. 2, three different porosity distribution patterns are considered in this paper for the shell's core including a uniform porosity distribution (UD) and two non-uniform ones (SI and SII). The elastic modulus of the FGP core varies along thickness direction as [34]

$$
\begin{array}{cc}
\text { UD: } & E_{c}=\left(1-e_{0}\right) E_{0}, \\
\text { SI }: & E_{c}(z)=\left[1-e_{1} \cos \left(\frac{\pi z}{h_{c}}\right)\right] E_{0}, \\
\text { SII } & E_{c}(z)=\left\{1-e_{2}\left[1-\cos \left(\frac{\pi z}{h_{c}}\right)\right]\right\} E_{0},
\end{array}
$$

where $E_{0}$ is the modulus of elasticity of the material with no porosity and $e_{0}, e_{1}$, and $e_{2}$ stand for the porosity parameters.

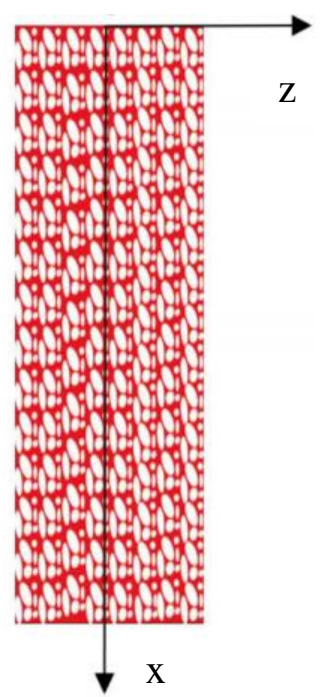

(a) UD

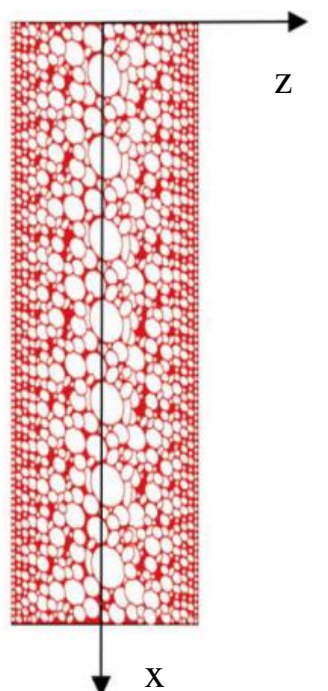

(b) SI

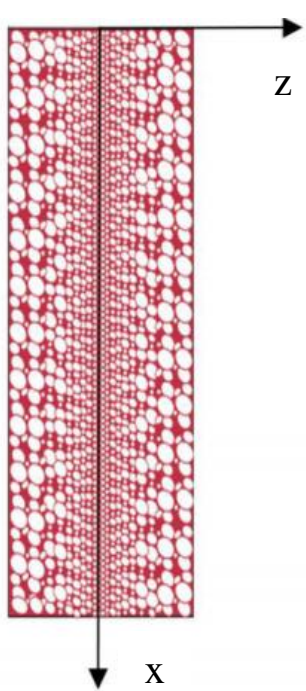

(c) SII

Fig. 2. Porosity distribution patterns [35]

To have a fair comparison between these porosity distribution patterns, it is better to regulate the porosity parameters to create the same value of mass. Utilizing the following equation between density $(\rho)$ and the elastic modulus [35]

$$
\frac{E_{c}(z)}{E_{0}}=\left[\frac{\rho_{c}(z)}{\rho_{0}}\right]^{2.73},
$$

in which $\rho_{0}$ represents the density of the material with no porosity, one can write

$$
\int_{0}^{\frac{h_{c}}{2}}\left(1-e_{0}\right)^{\frac{1}{2.73}} d z=\int_{0}^{\frac{h_{c}}{2}}\left[1-e_{1} \cos \left(\frac{\pi z}{h_{c}}\right)\right]^{\frac{1}{2.73}} d z
$$




$$
\int_{0}^{\frac{h_{c}}{2}}\left\{1-e_{2}\left[1-\cos \left(\frac{\pi z}{h_{c}}\right)\right]\right\}^{\frac{1}{2.73}} d z=\int_{0}^{\frac{h_{c}}{2}}\left[1-e_{1} \cos \left(\frac{\pi z}{h_{c}}\right)\right]^{\frac{1}{2.73}} d z .
$$

For some values of the porosity parameter $e_{1}$, the corresponding values of the porosity parameters $e_{0}$ and $e_{2}$ can be found in Table 1 and can be approximately stated as bellow [34]:

$$
\begin{aligned}
& e_{0}=1.944 e_{1}^{6}-3.417 e_{1}^{5}+2.278 e_{1}^{4}-0.6708 e_{1}^{3}+0.122 e_{1}^{2}+0.6362 e_{1}, \\
& e_{2}=-0.4269 e_{1}^{3}-0.009286 e_{1}^{2}+1.732 e_{1} .
\end{aligned}
$$

Table 1. Regulating the porosity parameters for various distributions patterns [35]

\begin{tabular}{cccccccc}
\hline$e_{1}$ & 0 & 0.1 & 0.2 & 0.3 & 0.4 & 0.5 & 0.6 \\
\hline$e_{0}$ & 0 & 0.0640 & 0.1287 & 0.1942 & 0.2609 & 0.3289 & 0.3988 \\
\hline$e_{2}$ & 0 & 0.1734 & 0.3426 & 0.5065 & 0.6637 & 0.8112 & 0.9432 \\
\hline
\end{tabular}

Based on Biot's poroelasticity theory for isotropic poroelastic mediums, the constitutive relations are stated as [16]

$$
\sigma_{i j}=2 G \varepsilon_{i j}+\lambda_{u} \varepsilon_{k k} \delta_{i j}-\alpha_{0} p \delta_{i j}, \quad i, j=1,2,3 .
$$

in which $\sigma_{\mathrm{ij}}$ and $\varepsilon_{\mathrm{ij}}$ sequentially stand for the stress and strain tensors and $\alpha_{0}$ is the Biot coefficient of effective stress. Also, with the following definitions, $G, \lambda \mathrm{u}, p, \varepsilon \mathrm{kk}$, and $\delta_{\mathrm{ij}}$ represent the shear modulus, undrained Lame parameter, pore fluid pressure, volumetric strain, and the Kronecker delta:

$$
G=\frac{E}{2(1+v)}, \quad \lambda_{u}=\frac{2 v_{u}}{1-2 v_{u}} G, \quad p=M\left(\xi-\alpha_{0} \varepsilon_{k k}\right), \quad \varepsilon_{k k}=\varepsilon_{x x}+\varepsilon_{\theta \theta}+\varepsilon_{z z}, \quad \delta_{i j}=\left\{\begin{array}{ll}
1 & i=j \\
0 & i \neq j
\end{array} .\right.
$$

in which $\xi$ stands for the variation of fluid volume content and $v_{\mathrm{u}}$ and $M$ sequentially represent undrained Poisson's ratio and Biot's modulus and are defined as below:

$$
v_{u}=\frac{3 v+\alpha_{0} B_{0}(1-2 v)}{3-\alpha_{0} B_{0}(1-2 v)}, \quad M=\frac{2\left(v_{u}-v\right)}{\alpha_{0}^{2}(1-2 v)\left(1-2 v_{u}\right)} G,
$$

where $B_{0}$ is known as the Skempton coefficient which deputizes the compressibility of the pore fluid. By increasing the Skempton coefficient from zero to one, the pore fluid varies from a completely compressible fluid to a noncompressible fluid.

For the undrained condition $(\xi=0)$, the plane stress assumption $\left(\sigma_{\mathrm{zz}}=0\right)$, and employing Eqs. (6) and (7), Eq. (5) can be stated in $x-\theta-z$ coordinates as below $\left(\gamma_{\mathrm{ij}}=2 \varepsilon_{\mathrm{ij}}\right)$ :

$$
\left\{\begin{array}{l}
\sigma_{x x} \\
\sigma_{\theta \theta} \\
\sigma_{\theta z} \\
\sigma_{x z} \\
\sigma_{x \theta}
\end{array}\right\}=\left[\begin{array}{ccccc}
C_{11} & C_{12} & 0 & 0 & 0 \\
C_{12} & C_{22} & 0 & 0 & 0 \\
0 & 0 & k_{s} C_{44} & 0 & 0 \\
0 & 0 & 0 & k_{s} C_{55} & 0 \\
0 & 0 & 0 & 0 & C_{66}
\end{array}\right]\left\{\begin{array}{l}
\varepsilon_{x x} \\
\varepsilon_{\theta \theta} \\
\gamma_{\theta z} \\
\gamma_{x z} \\
\gamma_{x \theta}
\end{array}\right\},
$$

where $k_{s}=5 / 6$ is known as the shear correction factor and 


$$
\begin{gathered}
C_{11}=C_{22}=2 f_{1} G, \quad C_{12}=2 f_{2} G, \quad C_{44}=C_{55}=C_{66}=G, \\
f_{1}=\frac{1-4 v+2 v_{u}}{1-3 v+2 v v_{u}}, \quad f_{2}=\frac{2(1-v) v_{u}-v}{1-3 v+2 v v_{u}} .
\end{gathered}
$$

For the face sheets $\left(e_{1}=e_{2}=e_{3}=0\right.$ and $\alpha_{0}=0$, and as a result $\left.v_{\mathrm{u}}=v\right)$, Eq. (9) can be stated as below:

$$
C_{11}=C_{22}=\frac{E_{f}}{1-v_{f}^{2}}, \quad C_{12}=v_{f} C_{11}, \quad C_{44}=C_{55}=C_{66}=G_{f}=\frac{E_{f}}{2\left(1+v_{f}\right)} .
$$

where the subscript $f$ represents the mechanical properties of the isotropic homogenous face sheets.

\subsection{Governing equations}

As stated in the FSDT, the following relation can be utilized for the displacement field [36]:

$$
\begin{aligned}
& u_{1}(x, \theta, z, t)=z \varphi_{x}(x, \theta, t)+u(x, \theta, t), \\
& u_{2}(x, \theta, z, t)=z \varphi_{\theta}(x, \theta, t)+v(x, \theta, t), \\
& u_{3}(x, \theta, z, t)=w(x, \theta, t) .
\end{aligned}
$$

in which $u_{1}, u_{2}$, and $u_{3}$ sequentially are the displacement components along of $x$, $\theta$, and $z$, directions; $u, v$, and $w$ show the corresponding components of displacement at the middle surface $(z=0)$; and $\varphi_{x}$ and $\varphi_{\theta}$ stand for the rotations about $\theta$ - and $x$ - axes, sequentially.

The non-zero components of the strain tensor $\left(\varepsilon_{\mathrm{ij}}\right)$ can be presented as below [36]:

$$
\begin{aligned}
& \varepsilon_{x x}=\frac{\partial u}{\partial x}+z \frac{\partial \varphi_{x}}{\partial x}, \quad \varepsilon_{\theta \theta}=\frac{\sin \alpha}{r} u+\frac{\cos \alpha}{r} w+\frac{1}{r} \frac{\partial v}{\partial \theta}+z\left(\frac{\sin \alpha}{r} \varphi_{x}+\frac{1}{r} \frac{\partial \varphi_{\theta}}{\partial \theta}\right), \\
& \gamma_{\theta z}=-\frac{\cos \alpha}{r} v+\frac{1}{r} \frac{\partial w}{\partial \theta}+\varphi_{\theta}, \quad \gamma_{x z}=\frac{\partial w}{\partial x}+\varphi_{x}, \\
& \gamma_{x \theta}=\frac{1}{r} \frac{\partial u}{\partial \theta}-\frac{\sin \alpha}{r} v+\frac{\partial v}{\partial x}+z\left(\frac{1}{r} \frac{\partial \varphi_{x}}{\partial \theta}-\frac{\sin \alpha}{r} \varphi_{\theta}+\frac{\partial \varphi_{\theta}}{\partial x}\right),
\end{aligned}
$$

The governing equations and boundary conditions can be derived utilizing Hamilton's principle as below [37]:

$$
\int_{t_{1}}^{t_{2}}\left(\delta W_{n . c .}+\delta T-\delta U_{s}\right) d t=0
$$

in which $\left[t_{1}, t_{2}\right]$ is an arbitrary time interval, $\delta$ shows the variational operator, $T$ shows kinetic energy, $U_{s}$ is strain energy and $W_{n . c}$. stands for the work done by external non-conservative loads. The shell's kinetic energy can be presented as below:

$$
T=\frac{1}{2} \iiint_{V} \rho\left[\left(\frac{\partial u_{1}}{\partial t}\right)^{2}+\left(\frac{\partial u_{2}}{\partial t}\right)^{2}+\left(\frac{\partial u_{3}}{\partial t}\right)^{2}\right] d V,
$$

where

$$
\iiint_{V}() d V=\iint_{S} \int_{-\frac{h}{2}}^{\frac{h}{2}}() d z d S
$$


in which $\mathrm{d} S=r \mathrm{~d} x \mathrm{~d} \theta$ shows the shell's surface.

Eq. (14) can be represented using Eqs. (11) and (15) as below:

$$
T=\frac{1}{2} \iint_{S}\left\{I_{0}\left[\left(\frac{\partial u}{\partial t}\right)^{2}+\left(\frac{\partial v}{\partial t}\right)^{2}+\left(\frac{\partial w}{\partial t}\right)^{2}\right]+2 I_{1}\left(\frac{\partial u}{\partial t} \frac{\partial \varphi_{x}}{\partial t}+\frac{\partial v}{\partial t} \frac{\partial \varphi_{\theta}}{\partial t}\right)+I_{2}\left[\left(\frac{\partial \varphi_{x}}{\partial t}\right)^{2}+\left(\frac{\partial \varphi_{\theta}}{\partial t}\right)^{2}\right]\right\} d S,
$$

where

$$
I_{i}=\int_{-\frac{h}{2}}^{\frac{h}{2}} \rho(z) z^{i} d z, \quad i=0,1,2 .
$$

Due to the symmetry in pore distribution patterns, it is evident that $I_{1}=0$, consequently, the variation of the shell's kinetic energy can be stated as below:

$$
\delta T=\iint_{S}\left[I_{0}\left(\frac{\partial u}{\partial t} \frac{\partial \delta u}{\partial t}+\frac{\partial v}{\partial t} \frac{\partial \delta v}{\partial t}+\frac{\partial w}{\partial t} \frac{\partial \delta w}{\partial t}\right)+I_{2}\left(\frac{\partial \varphi_{x}}{\partial t} \frac{\partial \delta \varphi_{x}}{\partial t}+\frac{\partial \varphi_{\theta}}{\partial t} \frac{\partial \delta \varphi_{\theta}}{\partial t}\right)\right] d S
$$

The variation of the shell's strain energy can be calculated as below

$$
\delta U_{s}=\iiint_{V} \sigma_{i j} \delta \varepsilon_{i j} d V
$$

which can be represented using Eqs. (12) and (15) as

$$
\begin{gathered}
\delta U_{s}=\iint_{S}\left(N_{x x} \frac{\partial \delta u}{\partial x}+\frac{N_{\theta \theta}}{r} \frac{\partial \delta v}{\partial \theta}+\frac{N_{\theta \theta} \sin \alpha}{r} \delta u+\frac{N_{\theta \theta} \cos \alpha}{r} \delta w+M_{x x} \frac{\partial \delta \varphi_{x}}{\partial x}+\frac{M_{\theta \theta} \sin \alpha}{r} \delta \varphi_{x}\right. \\
+\frac{M_{\theta \theta}}{r} \frac{\partial \delta \varphi_{\theta}}{\partial \theta}-\frac{Q_{\theta z} \cos \alpha}{r} \delta v+\frac{Q_{\theta z}}{r} \frac{\partial \delta w}{\partial \theta}+Q_{\theta z} \delta \varphi_{\theta}+Q_{x z} \frac{\partial \delta w}{\partial x}+Q_{x z} \delta \varphi_{x}+\frac{N_{x \theta}}{r} \frac{\partial \delta u}{\partial \theta} \\
\left.+N_{x \theta} \frac{\partial \delta v}{\partial x}-\frac{N_{x \theta} \sin \alpha}{r} \delta v+\frac{M_{x \theta}}{r} \frac{\partial \delta \varphi_{x}}{\partial \theta}+M_{x \theta} \frac{\partial \delta \varphi_{\theta}}{\partial x}-\frac{M_{x \theta} \sin \alpha}{r} \delta \varphi_{\theta}\right) d S,
\end{gathered}
$$

where

$$
\left\{\begin{array}{l}
N_{x x} \\
N_{\theta \theta} \\
N_{x \theta}
\end{array}\right\}=\int_{-\frac{h}{2}}^{\frac{h}{2}}\left\{\begin{array}{l}
\sigma_{x x} \\
\sigma_{\theta \theta} \\
\sigma_{x \theta}
\end{array}\right\} d z, \quad\left\{\begin{array}{l}
M_{x x} \\
M_{\theta \theta} \\
M_{x \theta}
\end{array}\right\}=\int_{-\frac{h}{2}}^{\frac{h}{2}}\left\{\begin{array}{l}
\sigma_{x x} \\
\sigma_{\theta \theta} \\
\sigma_{x \theta}
\end{array}\right\} z d z, \quad\left\{\begin{array}{l}
Q_{x z} \\
Q_{\theta z}
\end{array}\right\}=\int_{-\frac{h}{2}}^{\frac{h}{2}}\left\{\begin{array}{l}
\sigma_{x z} \\
\sigma_{\theta z}
\end{array}\right\} d z .
$$

By substituting Eqs. (3) and (4) into Eq. (23), the following equation can be achieved:

$$
\begin{aligned}
& N_{x x}=A_{11} \frac{\partial u}{\partial x}+\frac{A_{12} \sin \alpha}{r} u+\frac{A_{12}}{r} \frac{\partial v}{\partial \theta}+\frac{A_{12} \cos \alpha}{r} w+B_{11} \frac{\partial \varphi_{x}}{\partial x}+\frac{B_{12} \sin \alpha}{r} \varphi_{x}+\frac{B_{12}}{r} \frac{\partial \varphi_{\theta}}{\partial \theta}, \\
& N_{\theta \theta}=A_{12} \frac{\partial u}{\partial x}+\frac{A_{22} \sin \alpha}{r} u+\frac{A_{22}}{r} \frac{\partial v}{\partial \theta}+\frac{A_{22} \cos \alpha}{r} w+B_{12} \frac{\partial \varphi_{x}}{\partial x}+\frac{B_{22} \sin \alpha}{r} \varphi_{x}+\frac{B_{22}}{r} \frac{\partial \varphi_{\theta}}{\partial \theta}, \\
& M_{x x}=B_{11} \frac{\partial u}{\partial x}+\frac{B_{12} \sin \alpha}{r} u+\frac{B_{12}}{r} \frac{\partial v}{\partial \theta}+\frac{B_{12} \cos \alpha}{r} w+D_{11} \frac{\partial \varphi_{x}}{\partial x}+\frac{D_{12} \sin \alpha}{r} \varphi_{x}+\frac{D_{12}}{r} \frac{\partial \varphi_{\theta}}{\partial \theta}, \\
& M_{\theta \theta}=B_{12} \frac{\partial u}{\partial x}+\frac{B_{22} \sin \alpha}{r} u+\frac{B_{22}}{r} \frac{\partial v}{\partial \theta}+\frac{B_{22} \cos \alpha}{r} w+D_{12} \frac{\partial \varphi_{x}}{\partial x}+\frac{D_{22} \sin \alpha}{r} \varphi_{x}+\frac{D_{22}}{r} \frac{\partial \varphi_{\theta}}{\partial \theta}, \\
& N_{x \theta}=\frac{A_{66}}{r} \frac{\partial u}{\partial \theta}+A_{66} \frac{\partial v}{\partial x}-\frac{A_{66} \sin \alpha}{r} v+\frac{B_{66}}{r} \frac{\partial \varphi_{x}}{\partial \theta}+B_{66} \frac{\partial \varphi_{\theta}}{\partial x}-\frac{B_{66} \sin \alpha}{r} \varphi_{\theta}, \\
& M_{x \theta}=\frac{B_{66}}{r} \frac{\partial u}{\partial \theta}+B_{66} \frac{\partial v}{\partial x}-\frac{B_{66} \sin \alpha}{r} v+\frac{D_{66}}{r} \frac{\partial \varphi_{x}}{\partial \theta}+D_{66} \frac{\partial \varphi_{\theta}}{\partial x}-\frac{D_{66} \sin \alpha}{r} \varphi_{\theta},
\end{aligned}
$$




$$
\begin{aligned}
& Q_{\theta z}=A_{44}\left(-\frac{\cos \alpha}{r} v+\frac{1}{r} \frac{\partial w}{\partial \theta}+\varphi_{\theta}\right), \\
& Q_{x z}=A_{55}\left(\frac{\partial w}{\partial x}+\varphi_{x}\right),
\end{aligned}
$$

where $(\mathrm{i}, \mathrm{j}=1,2,6)$

$$
\left\{\begin{array}{l}
A_{i j} \\
B_{i j} \\
D_{i j}
\end{array}\right\}=\int_{-\frac{h}{2}}^{\frac{h}{2}} C_{i j}(z)\left\{\begin{array}{c}
1 \\
z \\
z^{2}
\end{array}\right\} d z, \quad\left\{\begin{array}{l}
A_{44} \\
A_{55}
\end{array}\right\}=k_{s} \int_{-\frac{h}{2}}^{\frac{h}{2}}\left\{\begin{array}{l}
C_{44}(z) \\
C_{55}(z)
\end{array}\right\} d z .
$$

Due to the symmetry in pore distribution patterns, it is evident that $B_{\mathrm{ij}}=0$, consequently, Eq. (22) can be simplified as follows:

$$
\begin{aligned}
& N_{x x}=A_{11} \frac{\partial u}{\partial x}+\frac{A_{12} \sin \alpha}{r} u+\frac{A_{12}}{r} \frac{\partial v}{\partial \theta}+\frac{A_{12} \cos \alpha}{r} w, \quad M_{x x}=D_{11} \frac{\partial \varphi_{x}}{\partial x}+\frac{D_{12} \sin \alpha}{r} \varphi_{x}+\frac{D_{12}}{r} \frac{\partial \varphi_{\theta}}{\partial \theta} \\
& N_{\theta \theta}=A_{12} \frac{\partial u}{\partial x}+\frac{A_{22} \sin \alpha}{r} u+\frac{A_{22}}{r} \frac{\partial v}{\partial \theta}+\frac{A_{22} \cos \alpha}{r} w, \quad M_{\theta \theta}=D_{12} \frac{\partial \varphi_{x}}{\partial x}+\frac{D_{22} \sin \alpha}{r} \varphi_{x}+\frac{D_{22}}{r} \frac{\partial \varphi_{\theta}}{\partial \theta}, \\
& N_{x \theta}=\frac{A_{66}}{r} \frac{\partial u}{\partial \theta}+A_{66} \frac{\partial v}{\partial x}-\frac{A_{66} \sin \alpha}{r} v, \quad M_{x \theta}=\frac{D_{66}}{r} \frac{\partial \varphi_{x}}{\partial \theta}+D_{66} \frac{\partial \varphi_{\theta}}{\partial x}-\frac{D_{66} \sin \alpha}{r} \varphi_{\theta}, \\
& Q_{\theta z}=A_{44}\left(-\frac{\cos \alpha}{r} v+\frac{1}{r} \frac{\partial w}{\partial \theta}+\varphi_{\theta}\right), \quad Q_{x z}=A_{55}\left(\frac{\partial w}{\partial x}+\varphi_{x}\right),
\end{aligned}
$$

In the free vibration analysis, the shell is not subjected to any external load $\left(\delta W_{n . c .}=0\right)$; consequently, by substituting Eqs. (18) and (20) into Eq. (13), the following set of the governing equations can be achieved:

$$
\begin{aligned}
& \frac{\partial N_{x x}}{\partial x}+\frac{\sin \alpha}{r}\left(N_{x x}-N_{\theta \theta}\right)+\frac{1}{r} \frac{\partial N_{x \theta}}{\partial \theta}-I_{0} \frac{\partial^{2} u}{\partial t^{2}}=0, \\
& \frac{1}{r} \frac{\partial N_{\theta \theta}}{\partial \theta}+\frac{\partial N_{x \theta}}{\partial x}+\frac{2 \sin \alpha}{r} N_{x \theta}+\frac{\cos \alpha}{r} Q_{\theta z}-I_{0} \frac{\partial^{2} v}{\partial t^{2}}=0, \\
& \frac{\partial Q_{x z}}{\partial x}+\frac{\sin \alpha}{r} Q_{x z}+\frac{1}{r} \frac{\partial Q_{\theta z}}{\partial \theta}-\frac{\cos \alpha}{r} N_{\theta \theta}-I_{0} \frac{\partial^{2} w}{\partial t^{2}}=0, \\
& \frac{\partial M_{x x}}{\partial x}+\frac{\sin \alpha}{r}\left(M_{x x}-M_{\theta \theta}\right)+\frac{1}{r} \frac{\partial M_{x \theta}}{\partial \theta}-Q_{x z}-I_{2} \frac{\partial^{2} \varphi_{x}}{\partial t^{2}}=0, \\
& \frac{1}{r} \frac{\partial M_{\theta \theta}}{\partial \theta}+\frac{\partial M_{x \theta}}{\partial x}+\frac{2 \sin \alpha}{r} M_{x \theta}-Q_{\theta z}-I_{2} \frac{\partial^{2} \varphi_{\theta}}{\partial t^{2}}=0,
\end{aligned}
$$

and the corresponding boundary condition can be stated as below:

Clamped(C): $\quad u=v=w=0, \quad \psi_{x}=\psi_{\theta}=0$,

$$
\begin{aligned}
& \text { Simply supported(S): } \quad N_{x x}=0, \quad v=w=0, \quad M_{x x}=0, \quad \psi_{\theta}=0, \\
& \text { Free(F): } \quad N_{x x}=N_{x \theta}=Q_{x z}=0, \quad M_{x x}=M_{x \theta}=0 .
\end{aligned}
$$

By substituting Eq. (24) into Eq. (25) and employing the following solution: 


$$
\left\{\begin{array}{l}
u(x, \theta, t) \\
v(x, \theta, t) \\
w(x, \theta, t) \\
\varphi_{x}(x, \theta, t) \\
\varphi_{\theta}(x, \theta, t)
\end{array}\right\}=\left\{\begin{array}{l}
U(x) e^{i \omega t} \cos (n \theta) \\
V(x) e^{i \omega t} \sin (n \theta) \\
W(x) e^{i \omega t} \cos (n \theta) \\
X(x) e^{i \omega t} \cos (n \theta) \\
\Theta(x) e^{i \omega t} \sin (n \theta)
\end{array}\right\}, \quad n=1,2,3, \mathrm{~K}
$$

in which $\omega$ is an eigenvalue and $n$ is called the circumferential wave number, the set of the governing equations can be represented as below:

$$
\begin{aligned}
& A_{11} U^{\prime \prime}+\frac{A_{11} \sin \alpha}{r} U^{\prime}-\frac{A_{22} \sin ^{2} \alpha+A_{66} n^{2}}{r^{2}} U+\frac{n\left(A_{12}+A_{66}\right)}{r} V^{\prime} \\
& -\frac{n\left(A_{22}+A_{66}\right) \sin \alpha}{r^{2}} V+\frac{A_{12} \cos \alpha}{r} W^{\prime}-\frac{A_{22} \sin 2 \alpha}{2 r^{2}} W+I_{0} \omega^{2} U=0, \\
& -\frac{n\left(A_{12}+A_{66}\right)}{r} U^{\prime}-\frac{n\left(A_{22}+A_{66}\right) \sin \alpha}{r^{2}} U+A_{66} V^{\prime \prime}+\frac{A_{66} \sin \alpha}{r} V^{\prime} \\
& -\frac{A_{22} n^{2}+A_{44} \cos ^{2} \alpha+A_{66} \sin ^{2} \alpha}{r^{2}} V-\frac{n\left(A_{22}+A_{44}\right) \cos \alpha}{r^{2}} W+\frac{A_{44} \cos \alpha}{r} \Theta+I_{0} \omega^{2} V=0, \\
& -\frac{A_{12} \cos \alpha}{r} U^{\prime}-\frac{A_{22} \sin 2 \alpha}{2 r^{2}} U-\frac{n\left(A_{22}+A_{44}\right) \cos \alpha}{r^{2}} V+A_{55} W^{\prime \prime}+\frac{A_{55} \sin \alpha}{r} W^{\prime} \\
& -\frac{A_{22} \cos { }^{2} \alpha+A_{44} n^{2}}{r^{2}} W+A_{55} X^{\prime}+\frac{A_{55} \sin \alpha}{r} X+\frac{n A_{44}}{r} \Theta+I_{0} \omega^{2} W=0, \\
& -A_{55} W^{\prime}+D_{11} X^{\prime \prime}+\frac{D_{11} \sin \alpha}{r} X^{\prime}-\left(A_{55}+\frac{D_{22} \sin ^{2} \alpha+D_{66} n^{2}}{r^{2}}\right) X \\
& +\frac{n\left(D_{12}+D_{66}\right)}{r} \Theta^{\prime}-\frac{n\left(D_{22}+D_{66}\right) \sin ^{2}}{r^{2}} \Theta+I_{2} \omega^{2} X=0, \\
& \frac{A_{44} \cos \alpha}{r} V+\frac{n A_{44}}{r} W-\frac{n\left(D_{12}+D_{66}\right)}{r} X^{\prime}-\frac{n\left(D_{22}+D_{66}\right) \sin \alpha}{r^{2}} X+D_{66} \Theta^{\prime \prime} \\
& +\frac{D_{66} \sin \alpha}{r} \Theta^{\prime}-\left(A_{44}+\frac{D_{22} n^{2}+D_{66} \sin ^{2} \alpha}{r^{2}}\right) \Theta+I_{2} \omega^{2} \Theta=0 .
\end{aligned}
$$

where prime indicates derivative with respect to the spatial variable $x$.

Likewise, by substituting Eq. (24) and (27) into Eq. (26), the boundary conditions can be stated as below:

Clamped(C): $U=V=W=0, \quad X=\Theta=0$,

Simply Supported $(\mathrm{S})$ :

$A_{11} U^{\prime}+\frac{A_{12} \sin \alpha}{r} U=0, \quad V=W=0, \quad D_{11} X^{\prime}+\frac{D_{12} \sin \alpha}{r} X=0, \quad \Theta=0$,

Free $(F)$ :

$$
\begin{aligned}
& A_{11} U^{\prime}+\frac{A_{12} \sin \alpha}{r} U+\frac{n A_{12}}{r} V+\frac{A_{12} \cos \alpha}{r} W=0, \quad-\frac{n}{r} U-\frac{\sin \alpha}{r} V+V^{\prime}=0, \\
& W^{\prime}+X=0, \quad D_{11} X^{\prime}+\frac{D_{12} \sin \alpha}{r} X+\frac{n D_{12}}{r} \Theta=0, \quad-\frac{n}{r} X+\Theta^{\prime}-\frac{\sin \alpha}{r} \Theta=0 .
\end{aligned}
$$




\section{Solution methodology}

In the current section, the DQM is employed as a well-accepted and well-known numerical approach to provide an approximate solution for the set of the governing equations (28) with any combination of the boundary conditions (29) at both ends of the shell $(x=0, L)$. Based on the main idea in the DQM, each derivative of a function like $P(x)$ can be estimated in terms of the weighted sum of its values at a set of grid points as below:

$$
\left\{\frac{d^{k} P}{d x^{k}}\right\}=\left[F^{(k)}\right]\{P\},
$$

where $\left[F^{(\mathrm{k})}\right]$ is called the weighting coefficient matrix related to the $k$ th order derivative and is presented as below [38]:

$$
\begin{gathered}
F_{i j}^{(1)}=\left\{\begin{array}{l}
\prod_{\substack{m=1 \\
m \neq i, j}}^{N}\left(x_{i}-x_{m}\right) \\
\prod_{\substack{m=1 \\
m \neq j}}^{N}\left(x_{j}-x_{m}\right) \\
\sum_{\substack{m=1 \\
m=i}}^{N}\left(x_{i}-x_{m}\right)^{-1}, i=j
\end{array} \quad i, j=1,2, \ldots, N,\right. \\
{\left[F^{(k)}\right]=\left[F^{(1)}\right]\left[F^{(k-1)}\right], \quad k=2,3, \mathrm{~K} .}
\end{gathered}
$$

The distribution pattern of the grid points plays an important role in the convergence of the solution in the DQM. With the following definition for $0 \leq x \leq L$, the Gauss-Lobatto-Chebyshev distribution pattern is utilized in this work [38]:

$$
x_{i}=\frac{L}{2}-\frac{L}{2} \cos \left(\frac{i-1}{N-1} \pi\right) .
$$

By applying Eq. (30) and the following notation:

$$
[A]=\left[F^{(1)}\right] \quad[B]=\left[F^{(2)}\right],
$$

the governing equations (28) can be stated as below:

$$
[K]\{c\}=\omega^{2}[M]\{c\},
$$

where

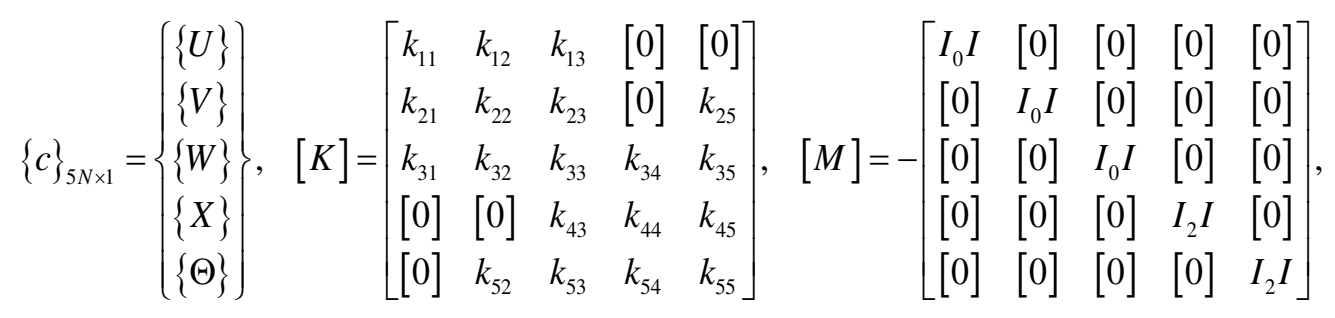




$$
\begin{aligned}
& k_{11}=A_{11}[B]+A_{11} \sin \alpha\left[a_{1}\right][A]-\left(A_{22} \sin ^{2} \alpha+A_{66} n^{2}\right)\left[a_{2}\right] I, \\
& k_{12}=n\left(A_{12}+A_{66}\right)\left[a_{1}\right][A]-n\left(A_{22}+A_{66}\right) \sin \alpha\left[a_{2}\right] I, \\
& k_{13}=A_{12} \cos \alpha\left[a_{1}\right][A]-0.5 A_{22} \sin 2 \alpha\left[a_{2}\right] I, \\
& k_{21}=-n\left(A_{12}+A_{66}\right)\left[a_{1}\right][A]-n\left(A_{22}+A_{66}\right) \sin \alpha\left[a_{2}\right], \\
& k_{22}=A_{66}[B]+A_{66} \sin \alpha\left[a_{1}\right][A]-\left(A_{22} n^{2}+A_{44} \cos ^{2} \alpha+A_{66} \sin ^{2} \alpha\right)\left[a_{2}\right], \\
& k_{23}=-n\left(A_{22}+A_{44}\right) \cos \alpha\left[a_{2}\right], \quad k_{25}=A_{44} \cos \alpha\left[a_{1}\right], \\
& k_{31}=-A_{12} \cos \alpha\left[a_{1}\right][A]-0.5 A_{22} \sin 2 \alpha\left[a_{2}\right], \quad k_{32}=-n\left(A_{22}+A_{44}\right) \cos \alpha\left[a_{2}\right], \\
& k_{33}=A_{55}[B]+A_{55} \sin \alpha\left[a_{1}\right][A]-\left(A_{22} \cos ^{2} \alpha+A_{44} n^{2}\right)\left[a_{2}\right], \\
& k_{34}=A_{55}[A]+A_{55} \sin \alpha\left[a_{1}\right], \quad k_{35}=n A_{44}\left[a_{1}\right], \\
& k_{43}=-A_{55}[A], \quad k_{44}=D_{11}[B]+D_{11} \sin \alpha\left[a_{1}\right][A]-A_{55} I-\left(D_{22} \sin ^{2} \alpha+D_{66} n^{2}\right)\left[a_{2}\right], \\
& k_{45}=n\left(D_{12}+D_{66}\right)\left[a_{1}\right][A]-n\left(D_{22}+D_{66}\right) \sin \alpha\left[a_{2}\right], \\
& k_{52}=A_{44} \cos \alpha\left[a_{1}\right], \quad k_{53}=n A_{44}\left[a_{1}\right], \\
& k_{54}=-n\left(D_{12}+D_{66}\right)\left[a_{1}\right][A]-n\left(D_{22}+D_{66}\right) \sin \alpha\left[a_{2}\right], \\
& k_{55}=D_{66}[B]+D_{66} \sin \alpha\left[a_{1}\right][A]-A_{44} I-\left(D_{22} n^{2}+D_{66} \sin ^{2} \alpha\right)\left[a_{2}\right] .
\end{aligned}
$$

in which $\left[\mathrm{a}_{1}\right]$ and $\left[\mathrm{a}_{2}\right]$ are two diagonal matrices defined as below:

$$
\left(a_{1}\right)_{i i}=\frac{1}{r_{i}}, \quad\left(a_{2}\right)_{i i}=\frac{1}{r_{i}^{2}} \text {. }
$$

By applying Eqs. (30) and (32) on Eq. (29), the boundary conditions can be presented as below:

$$
[\Gamma]\{c\}=\{0\}
$$

where

$$
[\Gamma]_{10 \times 5 N}=\left[\begin{array}{rrrrr}
\Gamma_{11} & \Gamma_{12} & \Gamma_{13} & \Gamma_{14} & \Gamma_{15} \\
\Gamma_{21} & \Gamma_{22} & \Gamma_{23} & \Gamma_{24} & \Gamma_{25} \\
\mathrm{M} & \mathrm{M} & \mathrm{M} & \mathrm{M} & \mathrm{M} \\
\Gamma_{101} & \Gamma_{102} & \Gamma_{103} & \Gamma_{104} & \Gamma_{105}
\end{array}\right],
$$

where $\Gamma_{11}-\Gamma_{55}$ are associated with the condition at $x=0$ and $\Gamma_{61}-\Gamma_{105}$ are associated with the condition at $x=L$.

For a truncated conical shell clamped at the small radius $(x=0)$ and simply supported at the large radius $(x=L)$ which is denoted by "CS" in this paper, $\Gamma_{11}-\Gamma_{105}$ are presented as below:

Clamped(C):

$$
\begin{aligned}
& \Gamma_{11}=\Gamma_{22}=\Gamma_{33}=\Gamma_{44}=\Gamma_{55}=I_{1}, \\
& \Gamma_{12}=\Gamma_{13}=\Gamma_{14}=\Gamma_{15}=\Gamma_{21}=\Gamma_{23}=\Gamma_{24}=\Gamma_{25}=\Gamma_{31}=\Gamma_{32}=\Gamma_{34} \\
& =\Gamma_{35}=\Gamma_{41}=\Gamma_{42}=\Gamma_{43}=\Gamma_{45}=\Gamma_{51}=\Gamma_{52}=\Gamma_{53}=\Gamma_{54}=\{0\}_{1 \times N},
\end{aligned}
$$


Simply supported(S):

$$
\begin{aligned}
& \Gamma_{61}=A_{11} A_{N}+\frac{A_{12} \sin \alpha}{b} I_{N}, \quad \Gamma_{94}=D_{11} A_{N}+\frac{D_{12} \sin \alpha}{b} I_{N}, \quad \Gamma_{72}=\Gamma_{83}=\Gamma_{105}=I_{N}, \\
& \Gamma_{62}=\Gamma_{63}=\Gamma_{64}=\Gamma_{65}=\Gamma_{71}=\Gamma_{73}=\Gamma_{74}=\Gamma_{75}=\Gamma_{81}=\Gamma_{82}=\Gamma_{84} \\
& =\Gamma_{85}=\Gamma_{91}=\Gamma_{92}=\Gamma_{93}=\Gamma_{95}=\Gamma_{101}=\Gamma_{102}=\Gamma_{103}=\Gamma_{104}=\{0\}_{1 \times N},
\end{aligned}
$$

where subscripts 1 and $N$ respectively stand for the first and last rows of each matrix.

Simultaneous solutions of Eqs. (34) and (37) generate an inequality between the numbers of the equations and unknown variables (non-square matrices in the final eigenvalue equation) [36]. To remove this inequality, let us divide the grid points into two sets: the boundary points ( $x_{1}$ and $\left.x_{\mathrm{N}}\right)$ and the domain ones $\left(x_{2}, x_{3}, \ldots, x_{N-2}, x_{N-1}\right)$. Ignoring the satisfiation of governing equations at the boundary points, Eq. (34) can be represented as below:

$$
\left[R^{\circ}\right]\{c\}=\omega^{2}[M 0]\{c\}
$$

in which the sign is utilized to show the created non-square matrices. By partitioning the matrices to separate the columns associated with the boundary and domain points, Eqs. (37) and (40) can be represented as below:

$$
\begin{aligned}
& {\left[K^{0}\right]_{d}\{c\}_{d}+\left[K^{0}\right]_{b}\{c\}_{b}=\omega^{2}\left(\left[M^{90}\right]_{d}\{c\}_{d}+\left[M^{0}\right]_{b}\{c\}_{b}\right),} \\
& {[\Gamma]_{b}\{c\}_{b}+[\Gamma]_{d}\{c\}_{d}=\{0\},}
\end{aligned}
$$

where subscripts " $b$ " and " $d$ " respectively show the boundary and domain points. By substituting Eq. (41-b) into Eq. (41-a), the following eigenvalue equation can be obtained:

$$
\left[K^{*}\right]\{c\}_{d}=\omega^{2}\left[M^{*}\right]\{c\}_{d}
$$

in which

$$
\left[K^{*}\right]=\left[K^{0}\right]_{b}[R]+\left[K^{0}\right]_{d}, \quad\left[M^{*}\right]=\left[M^{0}\right]_{b}[R]+\left[M^{0}\right]_{d}, \quad[R]=-[\Gamma]_{b}^{-1}[\Gamma]_{d} .
$$

Solving Eq. (42) results the shell's natural frequencies $(\omega)$. The natural frequencies in various vibrational modes are denoted by $\omega_{\mathrm{nm}}$ in which the first subscript $(n)$ is the circumferential wave number (Eq. (27)), and the second one $(m)$ is employed to indicate the meridional mode number. Also, the following definition is utilized in this paper to present the natural frequencies in a dimensionless form:

$$
\lambda_{n m}=\omega_{n m} a \sqrt{\frac{\rho_{f}}{E_{f}}} .
$$

where $\rho_{\mathrm{f}}$ and $E_{\mathrm{f}}$ sequentially stand for the density and elastic modulus of the face sheets.

\section{Numerical results}

Numerical results are presented this current part of the paper for the presented solution. In what follows, except as expressly stated, a CS conical shell is considered with the geometrical 
characteristics $a=0.5 \mathrm{~m}, \alpha=45^{\circ}, h / a=0.1, L / a=4$, and $h_{\mathrm{c}} / h=0.5$. The shell consists of an FGP core of distribution pattern SI, $e_{1}=0.5$, and $B_{0}=0.5$. The mechanical properties of the FGP core are $\rho 0=2700 \mathrm{~kg} / \mathrm{m}^{3}, v=0.25, E_{0}=60 \mathrm{GPa}$, and $\alpha_{0}=0.19[16,39]$ and those of the face sheets are $\rho_{f}=2707$ $\mathrm{kg} / \mathrm{m}^{3}, v_{f}=0.3$, and $E_{f}=70 \mathrm{GPa}$.

\subsection{Convergence analysis and validation}

The convergence analysis of the presented solution is examined in Fig. 3 for some vibrational modes. This figure shows that as the number of grid points grows ( $N$ in Eqs. (30)-(32)), values of the natural frequencies converge rapidly which approves the convergence analysis of the numerical solution performed in the meridional direction. In what follows, numerical examples are presented for $N=11$.

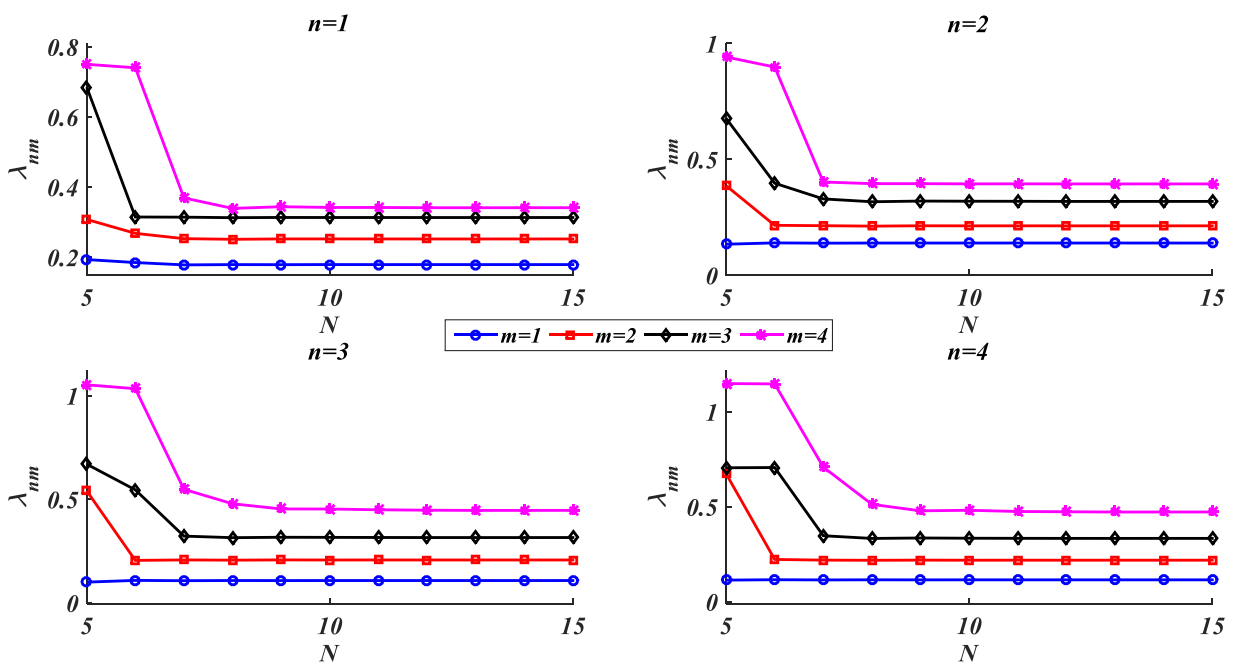

Fig. 3. Convergence analysis of the presented solution

Table 2. First dimensionless natural frequency $\left(\Omega_{\mathrm{n} 1}=\omega_{\mathrm{n} 1} b\left[\rho\left(1-v^{2}\right) / E\right]^{0.5}\right)$ of a homogenous isotropic truncated conical shell with various boundary conditions $\left(v=0.3, \alpha=45^{\circ}, L \sin \alpha / b=0.5, h / b=0.01\right)$

\begin{tabular}{ccccccccccc}
\hline & & $n=1$ & $n=2$ & $n=3$ & $n=4$ & $n=5$ & $n=6$ & $n=7$ & $n=8$ & $n=9$ \\
\hline \multirow{2}{*}{ CC } & Present & 0.8117 & 0.6694 & 0.5426 & 0.4563 & 0.4085 & 0.3956 & 0.4133 & 0.4555 & 0.5159 \\
& Liew et al. [40] & 0.8120 & 0.6696 & 0.5428 & 0.4565 & 0.4088 & 0.3961 & 0.4141 & 0.4567 & 0.5175 \\
\hline \multirow{2}{*}{ SC } & Present & 0.8115 & 0.6611 & 0.5246 & 0.4318 & 0.3825 & 0.3733 & 0.3979 & 0.4467 & 0.5115 \\
& Liew et al. [40] & 0.8113 & 0.6610 & 0.5244 & 0.4316 & 0.3832 & 0.3732 & 0.3980 & 0.4472 & 0.5124 \\
\hline \multirow{2}{*}{ CS } & Present & 0.7094 & 0.6473 & 0.5200 & 0.4159 & 0.3589 & 0.3445 & 0.3641 & 0.4082 & 0.4692 \\
& Liew et al. [40] & 0.7095 & 0.6473 & 0.5199 & 0.4158 & 0.3589 & 0.3446 & 0.3644 & 0.4088 & 0.4701 \\
\hline \multirow{2}{*}{ SS } & Present & 0.5460 & 0.6308 & 0.5062 & 0.3942 & 0.3339 & 0.3235 & 0.3507 & 0.4014 & 0.4661 \\
& Liew et al. [40] & 0.5462 & 0.6309 & 0.5061 & 0.3941 & 0.3337 & 0.3235 & 0.3510 & 0.4019 & 0.4671 \\
\hline
\end{tabular}


In order to check the precision of the presented solution, two examples are provided in this section. As the first example, consider an isotropic homogenous $(v=0.3)$ truncated conical shell of $\alpha=45^{\circ}$, $L \sin \alpha / b=0.5$, and $h / b=0.01$. For $m=1$ and various values of the circumferential wave number, dimensionless natural frequencies $\left(\Omega_{\mathrm{nm}}=\omega_{\mathrm{nm}} b\left[\rho\left(1-v^{2}\right) / E\right]^{0.5}\right)$ are tabulated in Table 2 versus those reported by Liew et al. [40]. As this table reveals, the results are in high matching which confirms the precision of the presented solution.

As the second example, consider an SS isotropic homogenous conical shell $(v=0.3)$ of $L \sin \alpha / b=0.25$ and $h / b=0.01$. For two selected values of the semi-vertex angle and various values of the circumferential wave number $(n=1,2, . ., 9)$, the dimensionless natural frequencies of the shell $\left(\Omega_{\mathrm{nm}}=\omega_{\mathrm{nm}} b\left[\rho\left(1-v^{2}\right) / E\right]^{0.5}\right)$ are presented for $m=1$ in Table 3 against those reported by Dai et al. [41]. This table confirms that results are in high matching which confirms the precision of the presented numerical solution.

Table 3. First dimensionless natural frequency $\left(\Omega_{\mathrm{n} 1}=\omega_{\mathrm{n} 1} b\left[\rho\left(1-v^{2}\right) / E\right]^{0.5}\right)$ of an SS isotropic homogenous truncated conical shell with various values of the semi-vertex angle $(v=0.3, L \sin \alpha / b=0.25, h / b=0.01)$

\begin{tabular}{cccccccccccc}
\hline & & $\mathrm{n}=1$ & $\mathrm{n}=2$ & $\mathrm{n}=3$ & $\mathrm{n}=4$ & $\mathrm{n}=5$ & $\mathrm{n}=6$ & $\mathrm{n}=7$ & $\mathrm{n}=8$ & $\mathrm{n}=9$ \\
\hline \multirow{2}{*}{$\alpha=30^{\circ}$} & Present & 0.5922 & 0.7908 & 0.7281 & 0.6348 & 0.5524 & 0.4940 & 0.4640 & 0.4628 & 0.4871 \\
& Dai et al. [41] & 0.5922 & 0.7909 & 0.7282 & 0.6349 & 0.5525 & 0.4941 & 0.4641 & 0.4633 & 0.4879 \\
\hline \multirow{2}{*}{$\alpha=60^{\circ}$} & Present & 0.4748 & 0.5715 & 0.5994 & 0.6044 & 0.6066 & 0.6144 & 0.6325 & 0.6627 & 0.7056 \\
& Dai et al. [41] & 0.4754 & 0.5721 & 0.6001 & 0.6053 & 0.6075 & 0.6156 & 0.6340 & 0.6646 & 0.7080 \\
\hline
\end{tabular}

\subsection{Parametric study}

The dependency of the shell's natural frequencies on the circumferential wave number is examined in Fig. 4. As this figure reveals, by increasing the circumferential wave number, the natural frequencies experience an initial reduction followed by increasing growth. In other words, there is a special value of the circumferential wave number which provides the lowest natural frequency (the fundamental frequency, $\lambda_{\mathrm{n} 1}$ ). Fig. 4 confirms that the boundary conditions can change this special value. 

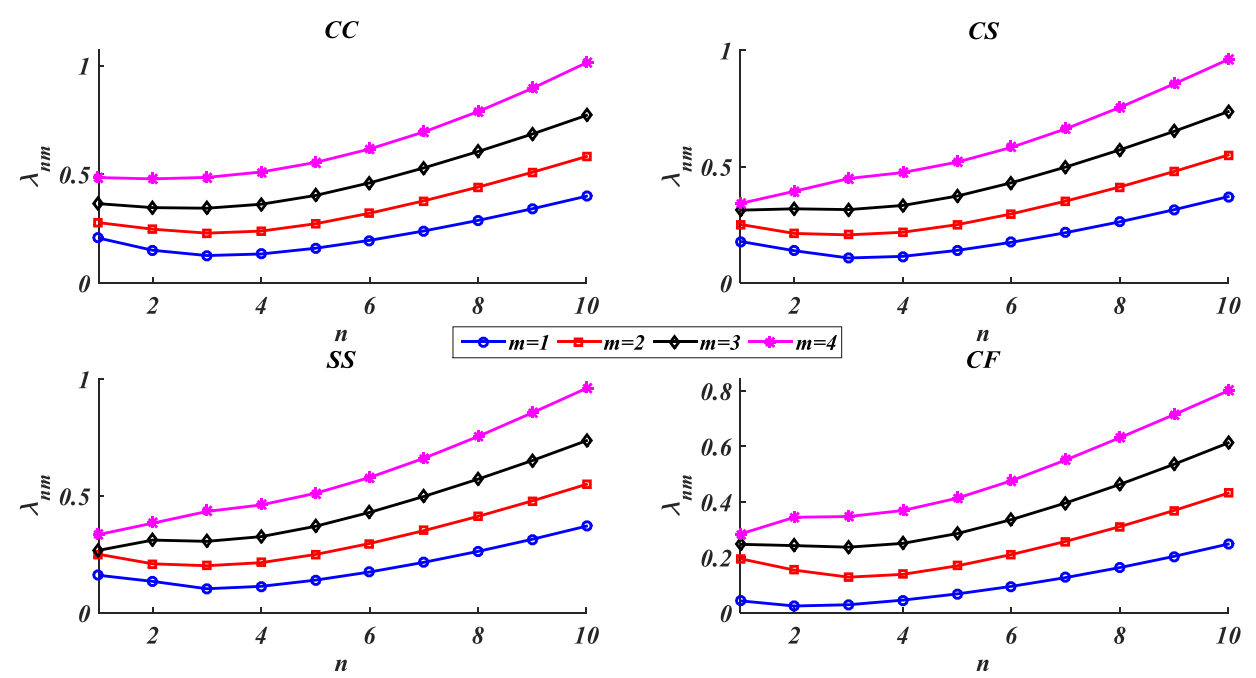

Fig. 4. Dependency of the natural frequencies on the circumferential wave number

Table 4 shows the influences of the boundary conditions on the shell's natural frequencies. As observed, the highest natural frequency at each vibrational mode belongs to the $\mathrm{CC}$ shell which means that the more constrained boundaries result in higher natural frequencies. A comparison between the CS, SC, and FC shells reveals that the natural frequencies of the SC shell are greater than the corresponding ones of the CS shell and in some vibrational modes, the natural frequency of the FC shell is greater than the natural frequency of the CS one. It means that the boundary condition at $x=L$ (the shell's larg radius) has a stronger effect on the natural frequencies of the conical shells rather than the boundary condition at $x=0$ (the shell's small radius).

Table 4. Dependency of the natural frequencies on the boundary conditions

\begin{tabular}{ccccccccc}
\hline \multicolumn{1}{c}{$n=1$} & \multicolumn{5}{c}{$n=2$} \\
\hline & CC & CS & SC & FC & CC & CS & SC & FC \\
\hline$m=1$ & $\mathbf{0 . 2 0 6 8}$ & 0.1788 & 0.2050 & 0.1955 & $\mathbf{0 . 1 5 0 5}$ & 0.1388 & 0.1461 & 0.1218 \\
$m=2$ & $\mathbf{0 . 2 7 7 7}$ & 0.2519 & 0.2726 & 0.2661 & $\mathbf{0 . 2 4 7 5}$ & 0.2130 & 0.2421 & 0.2047 \\
$m=3$ & $\mathbf{0 . 3 6 4 9}$ & 0.3131 & 0.3556 & 0.3338 & $\mathbf{0 . 3 4 6 6}$ & 0.3189 & 0.3370 & 0.2886 \\
$m=4$ & $\mathbf{0 . 4 8 4 9}$ & 0.3417 & 0.4627 & 0.4055 & $\mathbf{0 . 4 7 8 8}$ & 0.3943 & 0.4612 & 0.3914 \\
\hline \multicolumn{1}{c}{$n=3$} \\
\hline \multicolumn{1}{c}{ CC } & SC & FC & CC & CS & SC & FC \\
\hline$m=1$ & $\mathbf{0 . 1 2 6 3}$ & 0.1076 & 0.1222 & 0.1145 & $\mathbf{0 . 1 3 3 5}$ & 0.1146 & 0.1322 & 0.1316 \\
$m=2$ & $\mathbf{0 . 2 2 9 4}$ & 0.2070 & 0.2226 & 0.1944 & $\mathbf{0 . 2 3 8 7}$ & 0.2183 & 0.2345 & 0.2281 \\
$m=3$ & $\mathbf{0 . 3 4 3 9}$ & 0.3155 & 0.3331 & 0.2875 & $\mathbf{0 . 3 6 2 2}$ & 0.3333 & 0.3537 & 0.3314 \\
$m=4$ & $\mathbf{0 . 4 8 5 7}$ & 0.4491 & 0.4687 & 0.3983 & $\mathbf{0 . 5 1 0 4}$ & 0.4747 & 0.4965 & 0.4439 \\
\hline
\end{tabular}


Table 5 is presented to investigate the effect of the pore distribution pattern on the shell's natural frequencies. As this table shows, in most vibrational modes, the highest natural frequency belongs to the SI pattern. As depicted in Fig. 2, in the SI pattern the big pores are distributed close to the shell's neutral surface (SI) which leads to the minimum reduction in the shell's flexural rigidity. It is noteworthy that alongside the flexural rigidity, the rotational inertia ( $I_{2}$ in Eq. (17)) can be affected by the pore distribution pattern. Consequently, in some cases, the highest natural frequency belongs to the SII pattern which has the minimum rotational inertia.

Table 5. Dependency of the natural frequencies on the pore distribution pattern

\begin{tabular}{|c|c|c|c|c|c|c|}
\hline & \multicolumn{3}{|c|}{$n=1$} & \multicolumn{3}{|c|}{$n=2$} \\
\hline & UD & SI & SII & UD & SI & SII \\
\hline$m=1$ & 0.1781 & 0.1788 & 0.1796 & 0.1382 & 0.1388 & 0.1393 \\
\hline$m=2$ & 0.2509 & 0.2519 & 0.2524 & 0.2121 & 0.2130 & 0.2130 \\
\hline$m=3$ & 0.3120 & 0.3131 & 0.3149 & 0.3171 & 0.3189 & 0.3173 \\
\hline \multirow[t]{3}{*}{$m=4$} & 0.3399 & 0.3417 & 0.3408 & 0.3928 & 0.3943 & 0.3960 \\
\hline & \multicolumn{3}{|c|}{$n=3$} & \multicolumn{3}{|c|}{$n=4$} \\
\hline & UD & SI & SII & UD & SI & SII \\
\hline$m=1$ & 0.1070 & 0.1076 & 0.1072 & 0.1138 & 0.1146 & 0.1134 \\
\hline$m=2$ & 0.2059 & 0.2070 & 0.2061 & 0.2169 & 0.2183 & 0.2161 \\
\hline$m=3$ & 0.3135 & 0.3155 & 0.3128 & 0.3310 & $\mathbf{0 . 3 3 3 3}$ & 0.3293 \\
\hline$m=4$ & 0.4460 & 0.4491 & 0.4440 & 0.4713 & 0.4747 & 0.4683 \\
\hline
\end{tabular}

The dependency of the shell's natural frequencies on the porosity parameter is examined in Fig. 5. By growing the porosity parameter the size of the pore increases which decreases both the rigidity and inertia of the shell. Consequently, as the porosity parameter grows, depended on the vibrational mode, both increase or decrease in the natural frequency can be seen. As shown in this figure, due to the confrontation between the reductions in the rigidity and inertia of the shell, an increase in the porosity parameter has no remarkable effect on the natural frequencies. Thus, to make it possible to show the small variations of the natural frequencies in different vibrational modes, simultaneously, the following frequency parameter is defined:

$$
\Lambda_{n m}=\frac{\lambda_{n m}}{\left.\lambda_{n m}\right|_{e_{1}=0}} .
$$

Fig. 5 confirms that for $(n, m)=1,2,3,4$, by increasing the porosity parameter from zero to $e_{1}=0.6$, the maximum reduction and increase in the natural frequencies are less than 5\% and $1.5 \%$, respectively. 


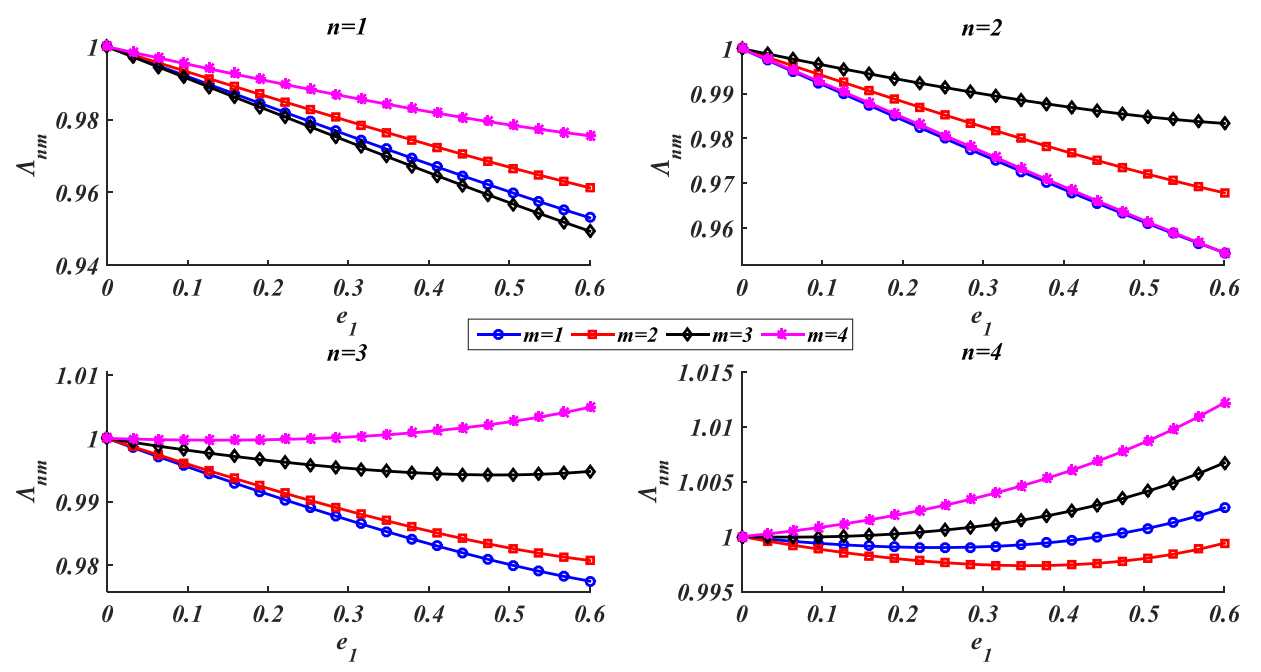

Fig. 5. Dependency of the natural frequencies on the porosity parameter

For a specified value of the shell's thickness, Fig. 6 shows the effect of the thickness of the FGP core on the shell's natural frequencies. By increasing the thickness of the FGP core, both inertia and rigidity of the shell decrease. Thus, as the thickness of the FGP core grows, depending on the vibrational mode, both increase or decrease in the natural frequency can be seen.

As this figure shows, due to the confrontation between the reductions in the inertia and rigidity of the shell, an increase in the shell's thickness has no considerable influence on the natural frequencies. Consequently, to make it possible to show the small variations of the natural frequencies in various vibrational modes, simultaneously, the frequency parameter is defined as below:
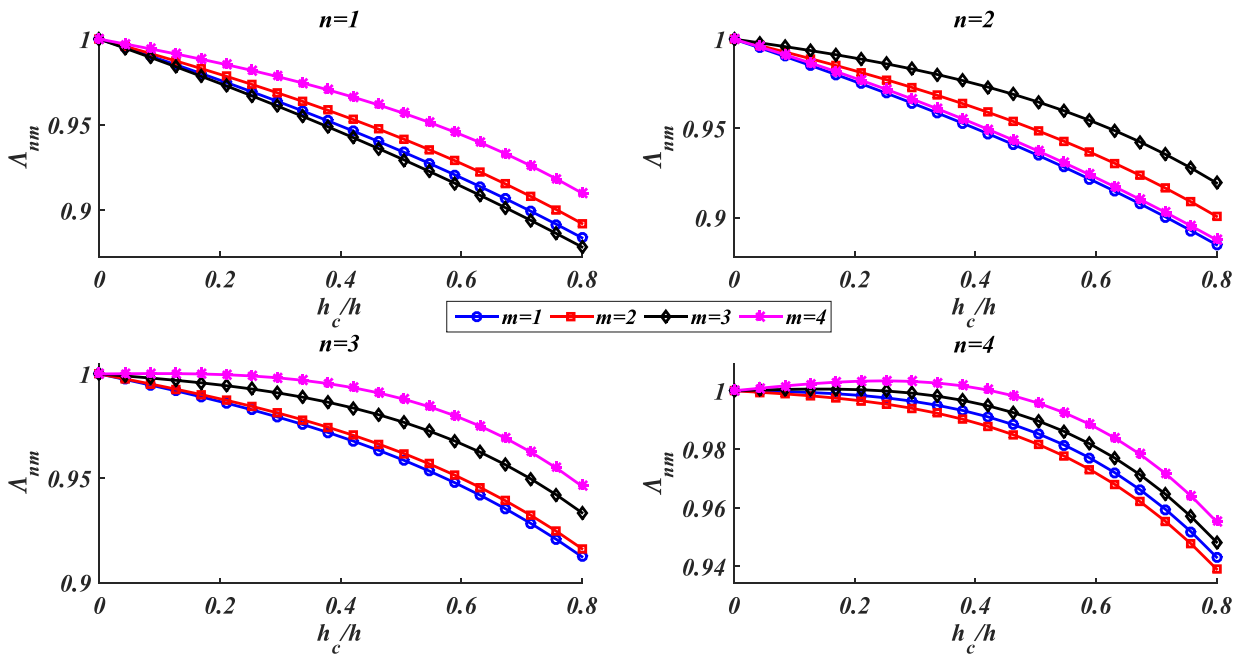

Fig. 6. Dependency of the natural frequencies on the thickness of the FGP core 


$$
\Lambda_{n m}=\frac{\lambda_{n m}}{\left.\lambda_{n m}\right|_{h_{c}=0}} .
$$

Fig. 6 shows that for $(n, m)=1,2,3,4$, by increasing the thickness of the FGP core from zero to $0.8 h$, the maximum reduction in the natural frequencies is less than $12 \%$.

Fig. 7 is provided to examine the effect of the compressibility of the pore fluid on the shell's natural frequencies. As observed, by increasing the Skempton parameter (decreasing the compressibility of the pore fluid), a small growth occurs in the natural frequencies. As this figure shows, for $(n, m)=1,2,3,4$, by increasing the Skempton parameter from the minimum possible value $\left(B_{0}=0\right)$ to the maximum possible value $\left(B_{0}=1\right)$, the maximum increase in the natural frequencies is less than $1 \%$.

It should be noticed that due to the weak effect of the compressibility of the pore fluid on the natural frequencies, to make it possible to show the small variations of the natural frequencies in different vibrational modes, simultaneously, the frequency parameter is defined as follows:

$$
\Lambda_{n m}=\frac{\lambda_{n m}}{\left.\lambda_{n m}\right|_{B_{0}=0}}
$$

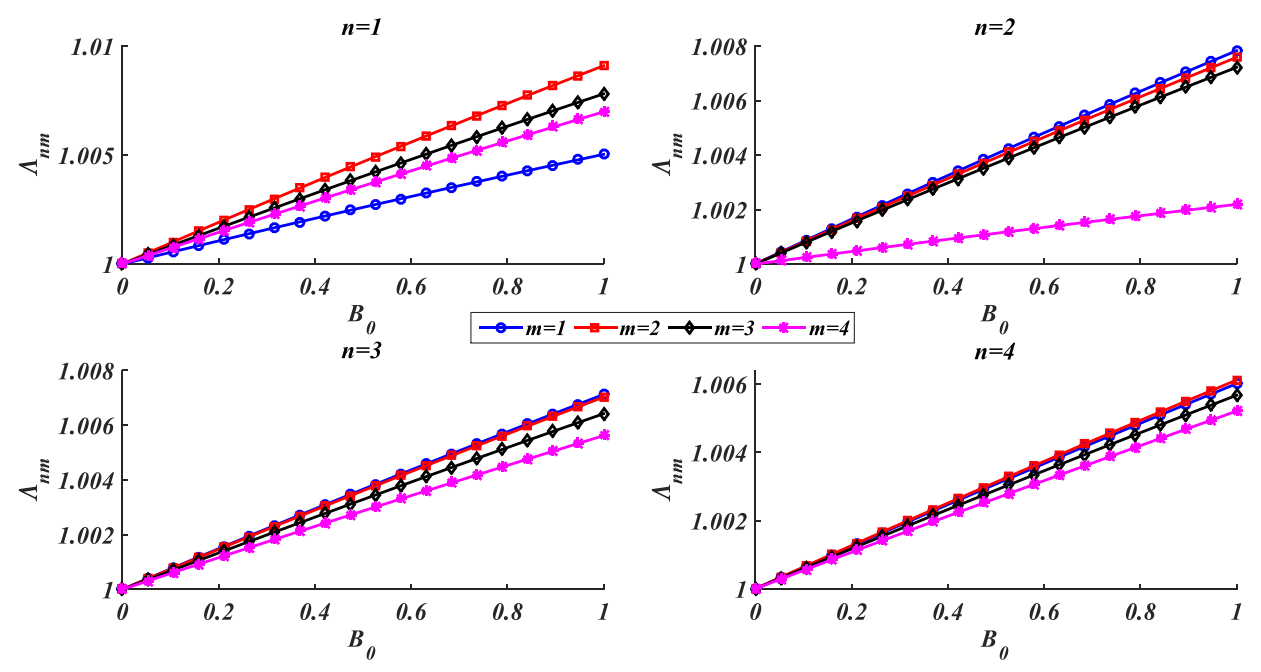

Fig. 7. Dependency of the natural frequencies on the compressibility of the pore fluid

\section{Conclusions}

The free vibrational analysis of a sandwich truncated conical shell with a saturated FGP core and two same homogenous isotropic face sheets was examined. The mechanical behavior of the saturated FGP core and mathematical modeling of the shell was performed based on Biot's theory and the FSDT respectively. Three different distribution patterns of the pores were investigated 
including a uniform distribution pattern and two non-homogenous symmetric ones. The main findings of the paper can be listed as below:

- The more constrained boundaries at the ends of the shell result in higher natural frequencies.

- The boundary condition at the shell's large radius has a stronger effect on the natural frequencies of the conical shells rather than the boundary condition at the shell's small radius.

- When the bigger pores are located close to the neutral surface of the shell, the natural frequencies become greater.

- $\quad$ By increasing the porosity parameter and thickness of the FGP core, both growth or reduction in the natural frequency can be seen. It depends on the vibrational mode.

- The lower compressibility of the pore fluid results in higher natural frequencies. But, the maximum increase is less than $1 \%$.

\section{References}

[1] Sofiyev A. On the vibration and stability behaviors of heterogeneous-CNTRC-truncated conical shells under axial load in the context of FSDT. Thin-Walled Structures. 2020;151:106747.

[2] Afshari H. Effect of graphene nanoplatelet reinforcements on the dynamics of rotating truncated conical shells. Journal of the Brazilian Society of Mechanical Sciences and Engineering. 2020;42:1-22.

[3] Zarei M, Rahimi G, Hemmatnezhad M. Free vibrational characteristics of grid-stiffened truncated composite conical shells. Aerospace Science and Technology. 2020;99:105717.

[4] Yousefi AH, Memarzadeh P, Afshari H, Hosseini SJ. Agglomeration effects on free vibration characteristics of three-phase CNT/polymer/fiber laminated truncated conical shells. Thin-Walled Structures. 2020;157:107077.

[5] Yousefi AH, Memarzadeh P, Afshari H, Hosseini SJ. Dynamic characteristics of truncated conical panels made of FRPs reinforced with agglomerated CNTs. Structures: Elsevier; 2021. p. 4701-17.

[6] Yousefi AH, Memarzadeh P, Afshari H, Hosseini SJ. Optimization of CNT/polymer/fiber laminated truncated conical panels for maximum fundamental frequency and minimum cost. Mechanics Based Design of Structures and Machines. 2021:1-23.

[7] Aris H, Ahmadi H. Nonlinear vibration analysis of FGM truncated conical shells subjected to harmonic excitation in thermal environment. Mechanics Research Communications. 2020;104:103499.

[8] Afshari H, Amirabadi H. Vibration characteristics of rotating truncated conical shells reinforced with agglomerated carbon nanotubes. Journal of Vibration and Control. 2021:10775463211000499.

[9] Damercheloo AR, Khorshidvand AR, Khorsandijou SM, Jabbari M. Free vibrational characteristics of GNP-reinforced joined conical-conical shells with different boundary conditions. Thin-Walled Structures. 2021;169:108287.

[10] Zhang C, Jin G, Wang Z, Sun Y. Dynamic stiffness formulation and vibration analysis of coupled conical-ribbed cylindrical-conical shell structure with general boundary condition. Ocean Engineering. 2021;234:109294.

[11] Fares M, Elmarghany MK, Atta D, Salem M. An improved layerwise formulation for free vibrations of multilayered FG truncated conical shells reinforced by carbon nanotubes. Composite Structures. 2021;275:114372.

[12] Li H, Hao Y, Zhang W, Liu L, Yang S, Wang D. Vibration analysis of porous metal foam truncated conical shells with general boundary conditions using GDQ. Composite Structures. 2021;269:114036.

[13] Singha TD, Rout M, Bandyopadhyay T, Karmakar A. Free vibration of rotating pretwisted FG-GRC sandwich conical shells in thermal environment using HSDT. Composite Structures. 2021;257:113144. 
[14] Adab N, Arefi M, Amabili M. A Comprehensive Vibration Analysis of Rotating Truncated Sandwich Conical Microshells Including Porous Core and GPL-Reinforced Face-sheets. Composite Structures. 2021:114761.

[15] Nasution MK, Syah R, Ramdan D, Afshari H, Amirabadi H, Selim MM, et al. Modeling and computational simulation for Supersonic flutter prediction of polymer/GNP/fiber laminated composite joined conical-conical shells. Arabian Journal of Chemistry. 2021:103460.

[16] Chen D, Kitipornchai S, Yang J. Nonlinear free vibration of shear deformable sandwich beam with a functionally graded porous core. Thin-Walled Structures. 2016;107:39-48.

[17] Mojahedin A, Jabbari M, Khorshidvand A, Eslami M. Buckling analysis of functionally graded circular plates made of saturated porous materials based on higher order shear deformation theory. Thin-Walled Structures. 2016;99:83-90.

[18] Biot MA. General theory of three-dimensional consolidation. Journal of applied physics. 1941;12:15564.

[19] Biot MA. Theory of elasticity and consolidation for a porous anisotropic solid. Journal of applied physics. 1955;26:182-5.

[20] Biot MA. General solutions of the equations of elasticity and consolidation for a porous material. J appl Mech. 1956;23:91-6.

[21] Biot MA. Theory of buckling of a porous slab and its thermoelastic analogy. Journal of Applied Mechanics. 1964;31:194-8.

[22] Leclaire P, Horoshenkov K, Cummings A. Transverse vibrations of a thin rectangular porous plate saturated by a fluid. Journal of Sound and Vibration. 2001;247:1-18.

[23] Shah SA. Axially symmetric vibrations of fluid-filled poroelastic circular cylindrical shells. Journal of Sound and Vibration. 2008;318:389-405.

[24] Kiani K, Avili HG, Kojorian AN. On the role of shear deformation in dynamic behavior of a fully saturated poroelastic beam traversed by a moving load. International Journal of Mechanical Sciences. 2015;94:84-95.

[25] Xiang Y, Jiang H, Lu J. Analyses of dynamic characteristics of a fluid-filled thin rectangular porous plate with various boundary conditions. Acta Mechanica Solida Sinica. 2017;30:87-97.

[26] Fouda N, El-Midany T, Sadoun A. Bending, buckling and vibration of a functionally graded porous beam using finite elements. Journal of Applied and Computational Mechanics. 2017;3:274-82.

[27] Mojahedin A, Jabbari M, Rabczuk T. Thermoelastic analysis of functionally graded porous beam. Journal of Thermal Stresses. 2018;41:937-50.

[28] Nikkhoo A, Tafakor R, Mofid M. An exact solution to the problems of flexo-poro-elastic structures rested on elastic beds acted upon by moving loads. Scientia Iranica. 2018.

[29] Enayat S, Hashemian M, Toghraie D, Jaberzadeh E. Bending, buckling and vibration analyses of FG porous nanobeams resting on Pasternak foundation incorporating surface effects. ZAMM-Journal of Applied Mathematics and Mechanics/Zeitschrift für Angewandte Mathematik und Mechanik. 2020:e202000231.

[30] Enayat S, Hashemian M, Toghraie D, Jaberzadeh E. A comprehensive study for mechanical behavior of functionally graded porous nanobeams resting on elastic foundation. Journal of the Brazilian Society of Mechanical Sciences and Engineering. 2020;42:1-24.

[31] Akbari H, Azadi M, Fahham H. Free vibration analysis of thick sandwich cylindrical panels with saturated FG-porous core. Mechanics Based Design of Structures and Machines. 2020:1-19.

[32] Akbari H, Azadi M, Fahham H. Flutter prediction of cylindrical sandwich panels with saturated porous core under supersonic yawed flow. Proceedings of the Institution of Mechanical Engineers, Part C: Journal of Mechanical Engineering Science. 2020:0954406220960786.

[33] Zhou X, Wang Y, Zhang W. Vibration and flutter characteristics of GPL-reinforced functionally graded porous cylindrical panels subjected to supersonic flow. Acta Astronautica. 2021;183:89-100.

[34] Khorshidvand AR, Damercheloo AR. Bending, axial buckling and shear buckling analyses of FGporous plates based on a refined plate theory. Australian Journal of Mechanical Engineering. 2021:1-20. 
[35] Wang YQ, Zhang ZY. Bending and buckling of three-dimensional graphene foam plates. Results in Physics. 2019;13:102136.

[36] Afshari H. Free vibration analysis of GNP-reinforced truncated conical shells with different boundary conditions. Australian Journal of Mechanical Engineering. 2020:1-17.

[37] Reddy JN. Energy principles and variational methods in applied mechanics: John Wiley \& Sons, 2017. [38] Bert CW, Malik M. Differential quadrature method in computational mechanics: a review. Applied Mechanics Reviews. 1996;49:1-28.

[39] Detournay E, Cheng AH-D. Fundamentals of poroelasticity. Analysis and design methods: Elsevier; 1993. p. 113-71.

[40] Liew KM, Ng TY, Zhao X. Free vibration analysis of conical shells via the element-free kp-Ritz method. Journal of Sound and Vibration. 2005;281:627-45.

[41] Dai Q, Cao Q, Chen Y. Frequency analysis of rotating truncated conical shells using the Haar wavelet method. Applied Mathematical Modelling. 2018;57:603-13. 